OPEN ACCESS

Edited by: Maura Pugliatti, University of Ferrara, Italy

Reviewed by:

Ozgur Kasapcopur, Istanbul University-Cerrahpasa, Turkey

Christos Bakirtzis,

Aristotle University of

Thessaloniki, Greece

*Correspondence:

Shigeki Arawaka shigeki.arawaka@ompu.ac.jp

Specialty section: This article was submitted to

Neuroepidemiology,

a section of the journal

Frontiers in Neurology

Received: 08 July 2021 Accepted: 15 November 2021 Published: 13 December 2021

Citation:

Sano E and Arawaka S (2021) White Matter Hyperintensities as a Risk Factor for Ischemic Stroke in Patients With Systemic Lupus Erythematosus. Front. Neurol. 12:738173. doi: 10.3389/fneur.2021.738173

\section{White Matter Hyperintensities as a Risk Factor for Ischemic Stroke in Patients With Systemic Lupus Erythematosus}

\section{Eri Sano and Shigeki Arawaka*}

Division of Neurology, Department of Internal Medicine IV, Faculty of Medicine, Osaka Medical and Pharmaceutical University, Takatsuki, Japan

Objective: The occurrence of ischemic stroke in patients with systemic lupus erythematosus (SLE) can cause extended periods of reduced daily activities. However, the risk factors for ischemic stroke in SLE patients are not fully elucidated. Herein, we examined the effect of white matter hyperintensities $(\mathrm{WMH})$ on the occurrence of ischemic stroke in SLE patients.

Methods: We analyzed the relationship between $\mathrm{WMH}$ burden and ischemic stroke using follow-up brain magnetic resonance imaging (MRI) data of 79 patients with SLE. Of these patients, 16 developed stroke during the observation period. $\mathrm{WMH}$ on MRI were classified into periventricular hyperintensities and deep white matter hyperintensities $(\mathrm{DWMH})$, while the lesion extent was graded using the Fazekas scale.

Results: Kaplan-Meier curves showed that ischemic stroke events were significantly associated with age at initial brain MRI of $\geq 40$ years $(p=0.015)$ and history of anti-phospholipid syndrome $(p=0.030)$. Additionally, ischemic stroke events were significantly associated with a one grade deterioration of periventricular hyperintensities $(p=0.003)$ and a one grade deterioration of DWMH $(p=0.002)$. Multivariate analysis using the logistic regression model showed that a one grade deterioration of DWMH was an independent risk factor for ischemic stroke (hazard ratio, 6.0; 95\% confidence interval, 1.3-27.4).

Conclusions: Although several factors affect the occurrence of ischemic stroke, SLE patients show increased risk of ischemic stroke via development of DWMH. An observation of DWMH deterioration on follow-up brain MRI may be useful for assessing the risk of ischemic stroke in SLE patients.

Keywords: systemic lupus erythematosus, white matter hyperintensity, ischemic stroke, risk factor, epidemiology 


\section{INTRODUCTION}

Systemic lupus erythematosus (SLE) is an autoimmune disease characterized by the production of autoantibodies, immune complex deposition, and multiple target organ damage (1). The occurrence of SLE varies considerably worldwide (2). In Japan, the prevalence of SLE ranges from 3.7 to 37.7 per 100,000 people. SLE mainly affects women in the $20-40$-year age group $(3,4)$. In an international cohort study, $40.3 \%$ of SLE patients showed neuropsychiatric symptoms during a mean observation period of $1.9 \pm 1.2$ years (5). Neurological manifestations of SLE include headache, cognitive impairment, myelopathy, and peripheral neuropathy (6). Additionally, cerebrovascular diseases often occur in SLE patients and cause a range of disturbances in daily living (7). For example, the risk of cerebral infarction in adult SLE patients was 2.18-fold higher than that in healthy individuals (8). However, the risk factors for occurrence of ischemic stroke in SLE patients are poorly characterized.

In the general population, white matter hyperintensities (WMH) are a common finding in brain magnetic resonance imaging (MRI) of adults $\geq 50$ years old (9). Furthermore, people $\geq 65$ years old show deterioration of WHM. The occurrence of WMH is correlated with aging, while vascular factors including hypertension, smoking, and diabetes mellitus can increase the risk for WMH (9). The presence of WMH also increases the risk of cerebrovascular diseases and cognitive impairment in the general population (10). A meta-analysis of prospective longitudinal studies in the general population reported that WMH on brain MRI increased the risk of ischemic stroke [hazard ratio (HR), 3.1; 95\% confidence interval (95\%CI), 2.3-4.1] (11). Although WMH are the most common brain MRI findings in SLE patients $(12,13)$, the influence of WMH on the occurrence of ischemic stroke in SLE patients remains unclear. In the present study, we investigated whether WMH were a risk factor for the occurrence of ischemic stroke in SLE patients.

\section{METHODS}

\section{Patients}

We analyzed 79 patients with SLE who visited Osaka Medical and Pharmaceutical University Hospital and underwent repeated brain MRI between April 2012 and June 2018. We enrolled patients with (1) age of $>16$ years, (2) a diagnosis according to the 1997 revised American College of Rheumatology classification criteria for SLE (14), and (3) no obvious evidence of any other disease that might explain the SLE-like symptoms. In the criteria, patients were required for fulfilling four or more criteria among clinical and immunological disorders. We excluded patients with (1) abnormalities on brain MRI (e.g., hydrocephalus, neoplasm, or metal deposition) or (2) history of brain trauma or operation. Only women were included in this study because the majority of patients at our facility were women. All patients had received an

Abbreviations: ANA, anti-nuclear antibodies; APS, anti-phospholipid syndrome; DWMH, deep white matter hyperintensities; HR, hazard ratio; IQR, interquartile range; MRI, magnetic resonance imaging; $\mathrm{PVH}$, periventricular hyperintensities; SLE, systemic lupus erythematosus; WMH, white matter hyperintensities. initial brain MRI for screening of brain lesions or for evaluating the cause of neuropsychiatric symptoms, and all patients had received more than one brain MRI in total (twice in 39 patients, three times in 11 patients, five times in seven patients, six times in five patients, seven times in four patients, and $>10$ times in four patients). A follow-up brain MRI was performed to evaluate the status of cerebrovascular diseases and other central nervous system lesions.

For diagnosis of anti-phospholipid syndrome (APS), we confirmed positivity for anti-phospholipid antibodies on two or more occasions that were at least 12 weeks apart and the presence of clinical histories (e.g., recurrent spontaneous abortion or thrombosis) (15). We defined positivity for antiphospholipid autoantibody as either anti-cardiolipin antibodies of $\geq 10.0$ $\mathrm{U} / \mathrm{ml}$, anti-cardiolipin- $\beta 2$-glycoprotein complex antibodies of $\geq 3.5 \mathrm{U} / \mathrm{ml}$, or a lupus anticoagulant of $\geq 1.3$. Clinical data were retrospectively retrieved from medical records. This study was conducted according to the 2013 Helsinki Declaration. The study protocol was approved by Osaka Medical and Pharmaceutical University Ethics Committee. The requirement for informed consent was waived because of the retrospective nature of the study and removal of all individual patient identifiers from the data (Approval number \# 609).

\section{Clinical Findings and Laboratory Examinations}

We collected baseline information, including age at SLE diagnosis and initial brain MRI, sex, vascular factors (history of smoking, diabetes mellitus, hypertension, and dyslipidemia), autoimmune abnormality (history of APS), and anti-platelet or anti-coagulant drug treatment for all patients. Smoking status was evaluated by interviews, and the patients were classified as current and past smokers or nonsmokers. Hypertension was defined with a resting blood pressure of $\geq 140 / 90 \mathrm{mmHg}$ or current use of antihypertensive agents (16). Diabetes mellitus was judged according to the American Diabetes Association criteria or by current medication history (17). Dyslipidemia was defined as low-density lipoprotein cholesterol levels of $\geq 160 \mathrm{mg} / \mathrm{dl}$, triglyceride levels of $\geq 150 \mathrm{mg} / \mathrm{dl}$, or by current medication history (18). Additionally, we collected baseline information on the titers of anti-nuclear antibodies (ANA), anti-double-stranded DNA antibodies, and the Systemic Lupus Erythematosus Disease Activity Index in measurable patients (19). The titers of ANA and anti-double-stranded DNA antibodies were measured by enzyme-linked immunosorbent assay.

\section{Assessment of WMH and Ischemic Stroke on Brain MRI}

Diffusion, T2, FLAIR, and T2*-weighted sequences (slice thickness, $2 \mathrm{~mm}$ ) were obtained using a 3.0T MRI (Signa HDxt, GE healthcare). To analyze WMH, the grades of periventricular hyperintensities $(\mathrm{PVH})$ and deep WMH $(\mathrm{DWMH})$ were evaluated using the Fazekas rating scale (20). PVH were evaluated on FLAIR-weighted transverse images covering the anterior horn and body of the lateral ventricles. DWMH were evaluated on FLAIR-weighted transverse images covering the semioval center. 
Initial brain MRI was used as the baseline image. To assess temporal changes in $\mathrm{WMH}$, we evaluated the differences in the grades of PVH and DWMH by subtracting the grades between baseline and the most recent MRI images. These data indicated a one grade deterioration of $\mathrm{PVH}$ and DWMH. Ischemic stroke was defined as acute onset of focal neurological deficits with abnormal high signal intensities on diffusion-weighted images and low apparent diffusion coefficient values on the apparent diffusion coefficient map.

\section{Statistical Analysis}

We analyzed the association between candidate factors and an ischemic stroke event from the initial brain MRI to the most recent MRI using Kaplan-Meier curves and the log-rank test. We estimated the HRs of the candidate factors for ischemic stroke by univariate analysis using the Cox proportional hazards model. Candidate factors were then subjected to multivariate analysis using the logistic regression model. Continuous values are expressed as median and interquartile range (IQR). A pvalue $<0.05$ was considered statistically significant. All statistical analyses were performed using statistical software (JMP Pro v14; SAS Institute Inc., Cary, NC, USA).

\section{RESULTS}

\section{Patient Characteristics}

We used clinical and laboratory data from 79 patients with SLE who underwent repeated brain MRI (Table 1). All patients were women. The median age at diagnosis was 31 years (IQR, 20-44 years), while the median age at initial brain MRI was 40 years (IQR, 31-53 years). Of these patients, 21 had APS (26.6\%) and 16 patients (20.3\%) had ischemic stroke as a new event after initial brain MRI. The median interval from initial brain MRI to the onset of ischemic stroke was 2 years (IQR, 2-10 years). At initial brain MRI, PVH were observed in 23 patients (29.1\%) and DWMH were observed in 30 patients (38.0\%). In follow-up brain MRI, a one grade deterioration of PVH and DWMH on the Fazekas scale was observed in 19 (24.1\%) and 24 (30.4\%) patients, respectively.

\section{Assessment of Clinical Factors Associated With Ischemic Stroke in Patients With SLE}

To explore the candidate factors associated with ischemic stroke in patients with SLE, we analyzed differences in the occurrence of ischemic stroke between patients with and without each factor using Kaplan-Meier curves with the log-rank test. We were unable to analyze the immunological data (e.g., the titers of ANA and anti-double-stranded DNA antibodies) or the Systemic Lupus Erythematosus Disease Activity Index scores because the number of measurable patients was small. Kaplan-Meier curves showed that ischemic stroke events were significantly associated with age at initial brain MRI of $\geq 40$ years $(p=0.015)$ and history of APS ( $p=0.03$ ) (Figure 1). Additionally, ischemic stroke events were significantly associated with a one grade deterioration of PVH $(p=0.003)$ and a one grade deterioration of DWMH $(p=0.002)$. Vascular factors, including history
TABLE 1 | Demographic data of 79 patients with Systemic Lupus Erythematous (SLE)

\begin{tabular}{|c|c|c|}
\hline & $n(\%)$ & $\begin{array}{l}\text { Median [Interquartile } \\
\text { range] }\end{array}$ \\
\hline Female & $79(100)$ & \\
\hline Age at diagnosis of SLE & & $31[20-44]^{\mathrm{a}}$ \\
\hline Age at initial brain MRI & & $40[31-53]^{\mathrm{a}}$ \\
\hline $\begin{array}{l}\text { Occurrence of ischemic } \\
\text { stroke }\end{array}$ & $16(20.3)$ & \\
\hline APS & $21(26.6)$ & \\
\hline $\begin{array}{l}\text { Current and past } \\
\text { smoker }\end{array}$ & $15(19.0)$ & \\
\hline Hypertension & $32(40.5)$ & \\
\hline Diabetes mellitus & 15 (19.0) & \\
\hline Dyslipidemia & $12(15.2)$ & \\
\hline $\begin{array}{l}\text { Anti-platelet drug } \\
\text { treatment }\end{array}$ & $25(31.6)$ & \\
\hline $\begin{array}{l}\text { Anti-coagulant drug } \\
\text { treatment }\end{array}$ & $4(5)$ & \\
\hline Titers of ANA & & $480[160-1280]^{b}$ \\
\hline $\begin{array}{l}\text { Titers of } \\
\text { anti-double-stranded } \\
\text { DNA antibodies }\end{array}$ & & $6.9[2.8-47.9]^{\mathrm{c}}$ \\
\hline SLEDAl & & $11.5[6-19.3]^{\mathrm{d}}$ \\
\hline $\mathrm{PVH}$ & $23(29.1)$ & \\
\hline DWMH & $30(38.0)$ & \\
\hline $\begin{array}{l}\text { One grade } \\
\text { deterioration of } \mathrm{PVH}\end{array}$ & $19(24.1)$ & \\
\hline $\begin{array}{l}\text { One grade } \\
\text { deterioration of DWMH }\end{array}$ & $24(30.4)$ & \\
\hline
\end{tabular}

$a_{n}=79 ;{ }^{b} n=36 ;{ }^{c} n=45 ;{ }^{d} n=30$. ANA, anti-nuclear antibodies; APS, antiphospholipid syndrome; DWMH, deep white matter hyperintensities; MRI, magnetic resonance imaging; PVH, periventricular hyperintensities; SLE, systemic lupus erythematosus; SLEDAl, systemic lupus erythematosus disease activity index.

of smoking, diabetes mellitus, hypertension, and dyslipidemia, showed no association with the ischemic stroke events in our SLE patients.

\section{Assessment of Clinical Factors Predictive for Ischemic Stroke in Patients With SLE}

To determine the predictive factors for the occurrence of ischemic stroke in patients with SLE, we analyzed clinical factors by univariate analysis using the Cox proportional hazards model (Table 2). The ischemic stroke events were associated with age at initial brain MRI (HR, 3.9; 95\%CI, 1.2-12.9) and history of APS (HR, 2.7; 95\%CI, 1.04-7.40). Furthermore, the ischemic stroke events were associated with a one grade deterioration of PVH (HR, 4.2; 95\%CI, 1.5-12.1) and a one grade deterioration of DWMH (HR, 6.0; 95\%CI, 1.7-21.3). Vascular factors, including history of smoking, diabetes mellitus, hypertension, and dyslipidemia, showed no significant association with the ischemic stroke events in our patients with SLE. Assessment of candidate factors by multivariate analysis using the logistic regression model showed that ischemic stroke events were only 

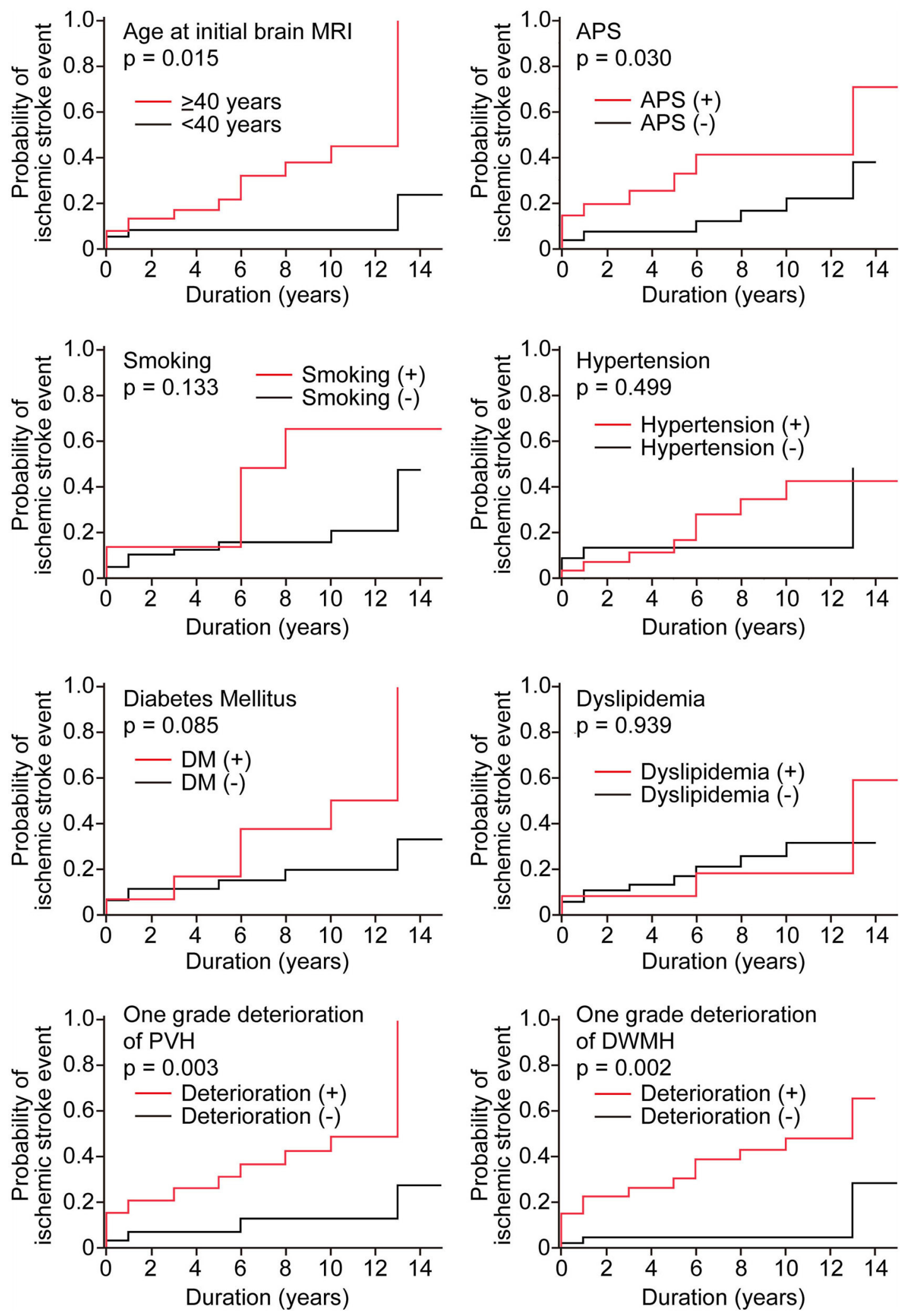

FIGURE 1 | Kaplan-Meier curves of ischemic stroke events in the study population disaggregated by the presence or absence of candidate risk factors. Kaplan-Meier curves were analyzed with the log-rank test. Duration represents the observation period from initial brain MRI to the most recent MRI. The red line shows the group with candidate factors, and the black line shows the group without candidate factors. In age, patients were classified into two groups $<40$ years (red line) and $\geq 40$ years (black line). 
TABLE 2 | Comparison of clinical factors predictive for ischemic stroke in patients with SLE.

\begin{tabular}{lcc}
\hline & $\begin{array}{c}\text { Univariate analysis } \\
\text { HR (95\% Cl) }\end{array}$ & $\begin{array}{c}\text { Multivariate analysis } \\
\text { HR (95\% Cl) }\end{array}$ \\
\hline Age at initial brain MRI & $3.9(1.2-12.9)$ & $2.9(0.6-13.5)$ \\
APS & $2.7(1.0-7.4)$ & $2.2(0.6-7.3)$ \\
Current and past smoker & $2.2(0.8-6.3)$ & $2.1(0.6-7.5)$ \\
Hypertension & $1.4(0.5-3.7)$ & $0.4(0.1-1.7)$ \\
Diabetes mellitus & $2.3(0.8-6.5)$ & $1.8(0.5-7.0)$ \\
Dyslipidemia & $0.9(0.3-3.1)$ & $0.7(0.2-2.7)$ \\
One grade deterioration of PVH & $4.2(1.5-12.1)$ & $1.2(0.3-5.1)$ \\
One grade deterioration of DWMH & $6.0(1.7-21.3)$ & $6.0(1.3-27.4)$ \\
\hline
\end{tabular}

APS, antiphospholipid syndrome; $\mathrm{Cl}$, confidence interval; DWMH, deep white matter hyperintensities; HR, hazard ratio; MRI, magnetic resonance imaging; $P V H$, periventricular hyperintensities.

Hazard ratios were analyzed by univariate analysis using the Cox proportional hazards model or multivariate analysis using the logistic regression model.

associated with a one grade deterioration of DWMH (HR, 6.0; 95\% CI, 1.3-27.4) (Table 2). These findings suggested that a one grade deterioration of DWMH was an independent risk factor for predicting ischemic stroke appearance in patients with SLE.

\section{DISCUSSION}

In the present study, we analyzed the risk factors for ischemic stroke in SLE patients to evaluate the influence of WMH burden on clinical events in the brain. In multivariate analysis using the logistic regression model, we identified a one grade deterioration of DWMH as an independent risk factor for the occurrence of ischemic stroke in patients with SLE. By contrast, a history of smoking, hypertension, diabetes mellitus, and dyslipidemia were not independent risk factors for this event. In the general population, these vascular factors are established as independent factors for ischemic stroke (21). The cause of this disparity between our patients with SLE and the general population is unclear. Previous study has demonstrated that hypertension is an independent risk factor for PVH and DWMH in patients with SLE (22). Thus, vascular factors may contribute to the occurrence of ischemic stroke via generation of WMH in patients with SLE, while the WMH burden may contribute to the occurrence of ischemic stroke. Our findings also suggest that therapeutic interventions designed to delay DWMH progression may prevent the occurrence of ischemic stroke in patients with SLE. Follow-up brain MRI to monitor DWMH deterioration may be useful for assessing ischemic stroke risk in patients with SLE.

Anti-phospholipid syndrome (APS) is often associated with SLE and is known to promote thrombosis in the cerebral arterial system, resulting in ischemic stroke and transient ischemic attack (23). However, in the present study, APS was not an independent risk factor for ischemic stroke in patients with SLE. The majority of our patients with APS had experienced an ischemic stroke before their initial MRI. Additionally, antiplatelet and/or anti-coagulant therapy had already been initiated in these patients. This preventive therapy may have reduced the risk of ischemic stroke during the observation period in patients with APS.

WMH are one of the main MRI features of small vessel disease in addition to acute lacunar infarcts or hemorrhages, lacunes as fluid-filled cavities of old infarcts, visible perivascular spaces, microbleeds, and brain atrophy (24). These changes are caused by variable etiologies, including arteriolosclerosis, cerebral amyloid angiopathy, and inflammatory and immunological causes (25). Pathologically, WMH are characterized by demyelination, astrogliosis, spondylosis, axonal loss, and widened perivascular spaces (10). It is difficult to address the etiology of WMH in SLE, although vascular factors are shown to be associated with WMH in SLE (22). To address this issue, further studies are required with detailed laboratory data (e.g., oligoclonal bands in the cerebrospinal fluid of SLE patients) and pathological examination of SLE brains.

In the present study, we examined changes in the brains of SLE patients using the same MRI conditions, which allowed us to assess WMH deterioration at follow-up brain MRI. However, there were some limitations in our study. First, this was a retrospective study. Second, the sample size of patients followed-up for analysis of ischemic stroke was small. Third, we were unable to assess the contribution of immunological factors to ischemic stroke because of a partial lack of immunological data. Finally, it is unclear whether the extent of WMH on the Fazekas scale is associated with ischemic stroke. Prospective large multicenter cohort studies are required to validate the contribution of $\mathrm{WMH}$ to the occurrence of ischemic stroke.

\section{CONCLUSIONS}

Patients with SLE have increased risk of ischemic stroke via development of DWMH. Follow-up brain MRI to monitor DWMH deterioration may be useful for assessing the risk of ischemic stroke.

\section{DATA AVAILABILITY STATEMENT}

The original contributions presented in the study are included in the article, further inquiries can be directed to the corresponding author.

\section{ETHICS STATEMENT}

The studies involving human participants were reviewed and approved by Osaka Medical and Pharmaceutical University Ethics Committee. Written informed consent for participation was not required for this study in accordance with the national legislation and the institutional requirements. 


\section{AUTHOR CONTRIBUTIONS}

All authors listed have made substantial, direct, and intellectual contribution to the work and approved it for publication.

\section{REFERENCES}

1. Dörner T, Furie R. Novel paradigms in systemic lupus erythematosus. Lancet. (2019) 393:2344-58. doi: 10.1016/S0140-6736(19)30546-X

2. Carter EE, Barr SG, Clarke AE. The global burden of SLE: prevalence, health disparities and socioeconomic impact. Nat Rev Rheumatol. (2016) 12:60520. doi: 10.1038/nrrheum.2016.137

3. Pons-Estel GJ, Alarcón GS, Scofield L, Reinlib L, Cooper GS. Understanding the epidemiology and progression of systemic lupus erythematosus. Semin Arthritis Rheum. (2010) 39:257-68. doi: 10.1016/j.semarthrit.2008.10.007

4. Tedeschi SK, Bermas B, Costenbader KH. Sexual disparities in the incidence and course of SLE and RA. Clin Immunol. (2013) 149:21118. doi: 10.1016/j.clim.2013.03.003

5. Hanly JG, Urowitz MB, Su L, Bae SC, Gordon C, Wallace DJ, et al. Prospective analysis of neuropsychiatric events in an international disease inception cohort of patients with systemic lupus erythematosus. Ann Rheum Dis. (2010) 69:529-35. doi: 10.1136/ard.2008.106351

6. Bertsias GK, Boumpas DT. Pathogenesis, diagnosis and management of neuropsychiatric SLE manifestations. Nat Rev Rheumatol. (2010) 6:35867. doi: 10.1038/nrrheum.2010.62

7. Hanly JG, Li Q, Su L, Urowitz MB, Gordon C, Bae SC, Romero-Diaz J, et al. Cerebrovascular events in systemic lupus erythematosus: results from an international inception cohort study. Arthritis Care Res. (2018) 70:147887. doi: 10.1002/acr.23509

8. Yazdany J, Pooley N, Langham J, Nicholson L, Langham S, Embleton N, et al. Systemic lupus erythematosus; stroke and myocardial infarction risk: a systematic review and meta-analysis. RMD Open. (2020) 6:e001247. doi: 10.1136/rmdopen-2020-001247

9. Habes M, Erus G, Toledo JB, Zhang T, Bryan N, Launer LJ, et al. White matter hyperintensities and imaging patterns of brain ageing in the general population. Brain. (2016) 139:1164-79. doi: 10.1093/brain/aww008

10. Prins ND, Scheltens $P$. White matter hyperintensities, cognitive impairment and dementia: an update. Nat Rev Neurol. (2015) 11:157-65. doi: 10.1038/nrneurol.2015.10

11. Debette S, Markus HS. The clinical importance of white matter hyperintensities on brain magnetic resonance imaging: systematic review and meta-analysis. BMJ. (2010) 341:c3666. doi: 10.1136/bmj.c3666

12. Sarbu N, Alobeidi F, Toledano P, Espinosa G, Giles I, Rahman A, et al. Brain abnormalities in newly diagnosed neuropsychiatric lupus: systematic MRI approach and correlation with clinical and laboratory data in a large multicenter cohort. Autoimmun Rev. (2015) 14:1539. doi: 10.1016/j.autrev.2014.11.001

13. Kaichi Y, Kakeda S, Moriya J, Ohnari N, Saito K, Tanaka Y, et al. Brain MR findings in patients with systemic lupus erythematosus with and without antiphospholipid antibody syndrome. AJNR Am J Neuroradiol. (2014) 35:1005. doi: 10.3174/ajnr.A3645

14. Hochberg MC. Updating the American College of Rheumatology revised criteria for the classification of systemic lupus erythematosus. Arthritis Rheum. (1997) 40:1725. doi: 10.1002/art.1780400928

15. Miyakis S, Lockshin MD, Atsumi T, Branch DW, Brey RL, Cervera R, et al. International consensus statement on an update of the classification criteria for definite antiphospholipid syndrome (APS). J Thromb Haemost. (2006) 4:295-306. doi: 10.1111/j.1538-7836.2006.01753.x

\section{ACKNOWLEDGMENTS}

The authors thank Tohru Takeuchi for aiding patient acquisition and Yuri Ito and Daisuke Nishioka for supporting statistical analysis.

16. Bakris G, Ali W, Parati G. ACC/AHA versus ESC/ESH on hypertension guidelines: JACC guideline comparison. J Am Coll Cardiol. (2019) 73:301826. doi: 10.1016/j.jacc.2019.03.507

17. Gabir MM, Hanson RL, Dabelea D, Imperatore G, Roumain J, Bennett PH, et al. The 1997 American Diabetes Association and 1999 World Health Organization criteria for hyperglycemia in the diagnosis and prediction of diabetes. Diabetes Care. (2000) 23:1108-12. doi: 10.2337/diacare.23.8.1108

18. Nakashima Y. Treatment of hyperlipidemia. Intern Med. (2000) 39:76572. doi: 10.2169/internalmedicine.39.765

19. Griffiths B, Mosca M, Gordon C. Assessment of patients with systemic lupus erythematosus and the use of lupus disease activity indices. Best Pract Res Clin Rheumatol. (2005) 19:685-708. doi: 10.1016/j.berh.2005.03.010

20. Fazekas F, Chawluk JB, Alavi A, Hurtig HI, Zimmerman RA. MR signal abnormalities at $1.5 \mathrm{~T}$ in Alzheimer's dementia and normal aging. AJR Am J Roentgenol. (1987) 149:351-6. doi: 10.2214/ajr.149.2.351

21. Eikelboom JW, Hankey GJ, Anand SS, Lofthouse E, Staples N, Baker RI. Association between high homocyst(e)ine and ischemic stroke due to largeand small-artery disease but not other etiologic subtypes of ischemic stroke. Stroke. (2000) 31:1069-75. doi: 10.1161/01.str.31.5.1069

22. Sano E, Ishida S, Takeuchi T, Nakaya Y, Arawaka S. Occurrence and Progression of White Matter Hyperintensities in Middle-aged Patients with Systemic Lupus Erythematosus. Bulletin of Osaka Medical and Pharmaceutical University. Available online at: https://www.ompu.ac.jp/ research/omc/outcome/magazine/bulletin/2021.html (accessed October 23, 2021).

23. Fanouriakis A, Kostopoulou M, Alunno A, Aringer M, Bajema I, Boletis JN, et al. 2019 update of the EULAR recommendations for the management of systemic lupus erythematosus. Ann Rheum Dis. (2019) 78:736-45. doi: 10.1136/annrheumdis-2019-215089

24. Wardlaw JM, Smith C, Dichgans M. Mechanisms of sporadic cerebral small vessel disease: insights from neuroimaging. Lancet Neurol. (2013) 12:48397. doi: 10.1016/S1474-4422(13)70060-7

25. Pantoni L. Cerebral small vessel disease: from pathogenesis and clinical characteristics to therapeutic challenges. Lancet Neurol. (2010) 9:689701. doi: 10.1016/S1474-4422(10)70104-6

Conflict of Interest: The authors declare that the research was conducted in the absence of any commercial or financial relationships that could be construed as a potential conflict of interest.

Publisher's Note: All claims expressed in this article are solely those of the authors and do not necessarily represent those of their affiliated organizations, or those of the publisher, the editors and the reviewers. Any product that may be evaluated in this article, or claim that may be made by its manufacturer, is not guaranteed or endorsed by the publisher.

Copyright (C) 2021 Sano and Arawaka. This is an open-access article distributed under the terms of the Creative Commons Attribution License (CC BY). The use, distribution or reproduction in other forums is permitted, provided the original author(s) and the copyright owner(s) are credited and that the original publication in this journal is cited, in accordance with accepted academic practice. No use, distribution or reproduction is permitted which does not comply with these terms. 